\title{
Laser Velocimetry Measurements in a Double Volute Centrifugal Pump
}

\author{
WILLIAM DE OJEDA, Graduate Research Assistant \\ Formerly at University of Virginia, Currently at Illinois Institute of Technology, Chicago, IL \\ RONALD D. FLACK, Professor and Chair \\ Department of Mechanical, Aerospace, and Nuclear Engineering, Rotating Machinery and Control Laboratory \\ University of Virginia, Charlottesville, VA 22903-2442 \\ STEVEN M. MINER, Associate Professor \\ Department of Mechanical Engineering U.S. Naval Academy Annapolis, MD 21402
}

(Received April 13, 1994; in final form March 31, 1995)

\begin{abstract}
Laser velocimetry measurements were taken in a double volute/single discharge centrifugal pump $(0.60$ specific speed, 1583 US units) with symmetrical volute halves. Blade-to-blade radial and tangential velocity profiles at the impeller exit are presented and compared to data for a similar single volute pump. Flow rates ranged from $40 \%$ of design flow to the design point. The blade-to-blade profiles were more uniform than for the single volute pump. Also, the average circumferential variations for the double volute pump were more symmetric than for the single volute pump. For the double volute geometry measurements indicate that radial inward flow (recirculation) was only present for flow rates below $60 \%$ of design flow, compared to $80 \%$ of design flow for the single volute pump. Velocity data was also used to determine volute losses, slip factor, and momentum contributions to the impeller radial forces. Volute losses were quantified and shown to increase for flow rates below $80 \%$ of design flow and were approximately $10 \%$ of the developed head at $40 \%$ flow. The efficiency in the double volute compared to the single volute shows decreased performance for flows above $55 \%$ of design flow, which is attributed to increased boundary layer friction; at low flow rates increased performance is ascribed to better control over the recirculation regions. Slip factors were symmetric around the volute but were lower than for a single volute pump. Finally, momentum contributions to the total impeller radial load were shown to be maximum at the design point, contributing $40 \%$ of the force developed by the pressure distribution; the significance diminished at lower flow rates and the contribution was negligible at $40 \%$ of the design flow.
\end{abstract}

Key Words: Turbomachinery; Pump; Volute; Impeller; Radial thrust; Laser velocimetry

$\mathbf{T}_{\mathrm{t}}^{\mathrm{s}, \mathrm{sen}}$ he reliability of centrifugal pumps is often coupled to reducing shaft deflections. Hydraulic forces on a pump impeller cause the shaft to deflect creating excessive metallic contact between it and wearing rings and bearings, and hence induce wear and leakage. Increased deflections yield additional fatigue which have been known to cause shaft failure.

This hydraulic force arises from non-symmetries in the flow field due to non-uniformity in both the static pressure and exit velocity distribution around the impel- ler discharge. Hydraulic forces are minimum when operating at design conditions although they may increase by several orders of magnitude at off-design flow. Pumps, even though intended to operate at design conditions, invariably function at low flow rates since this range covers start-up and shut-down. Furthermore, operation at low flow rates may represent continuous operation for many machines that are required to yield higher discharge pressures or lower flow rates than originally intended. A number of researchers have exam- 
ined impeller forces as reviewed by de Ojeda et al. (1992). For example, Guinzburg et al. (1992), Adkins and Brennen (1988), Jery et al. (1985), and Ohashi et al. (1981) all directly measured the radial thrust loads on impellers.

A few papers have concentrated on double volute pumps. Such a configuration typically decreases the hydraulic force upon the impeller by creating a more symmetric flow field in the volute. However, the increased surface area typically reduces pump performance because of the increased shear loss in the added boundary layers. Thus, for a double volute pump design the trade-off is improved reliability (because of lower thrusts) in exchange for minor drops in efficiency and performance head.

Howard et al. (1987) measured pressures and used flow visualization in three laboratory pumps. One was a single volute and the others were double volute/single discharge. They noted the effects of volute geometry on both local pressure fields in the impeller and volute. Pressures were strongly dependent on the circumferential position. Kanki et al. (1981) used pressure sensors on two impellers in a double volute/single discharge pump. They measured static and fluctuating pressures for concentric and eccentric impellers. At $100 \%$ of design flow they measured a nearly uniform pressure distribution in the volute. At $17 \%$ flow (or $17 \%$ capacity) very low pressures at the tongues were seen. They also directly measured forces using an overhung rotor with strain gages mounted to the shaft. Agostinelli et al. (1960) tested 16 different pumps with different specific speeds. Single and double volute/single discharge pumps were tested. Calibrated strain gages on the bearing housing were used to measure two components of force. Results are presented for flow rates from 0 to $170 \%$ of design conditions. The thrust proportionality factor originally proposed by Stepanoff as a function of capacity was revised by the authors to also be a function of specific speed.

The double volute pump, which is examined here, was earlier tested as a single volute/single discharge unit by Hamkins and Flack (1987), Beaudoin et al. (1992), and others. De Ojeda et al. (1992) modified this pump to operate as a double volute/single discharge pump. The pressure measurements around the impeller periphery and along the casing wall were documented for the pump assembled with a single volute and with two versions of a double volute. One version had a splitter located mid-way between the impeller and volute case. The second version had a log spiral splitter that matched the volute shape; for this geometry the two passages around the impeller were identical. These measurements yielded the pressure component of the radial thrust on the impeller. The second double volute version demonstrated the lowest pressure induced radial thrust on the impeller; at low flow rates the impeller force was less than half of the thrust for the first version.

In the current paper velocity data from non-intrusive laser velocimetry around the impeller periphery are documented. The velocity data is used to indicate the basic internal flow differences between the double volute pump and a single volute pump. These velocities are also used to calculate the momentum flux component of the radial thrust. Only the log spiral splitter version of the double volute pump is studied herein. In addition to calculating the total radial thrust upon the impeller, the pressure and velocity data are used to examine the effect of the double volute on pump performance. Impeller slip factors and losses in the volute are also reported.

\section{EXPERIMENTAL APPARATUS}

\section{Pump and Flow Loop}

The pump used for this study is documented in Hamkins and Flack (1987), Miner et al. (1989), and de Ojeda et al. (1992). The impeller passage width, b, and peripheral diameter, D, are 24.6 and $203.2 \mathrm{~mm}$, respectively. The running speed, $\omega$, for the pump is $620 \mathrm{RPM}$. The design point of the pump is $6.3 \mathrm{l} / \mathrm{s}$ at $22.2 \mathrm{kPa}$ total head. The design point is based on an estimate of the impellervolute matching point, the condition which causes the average angular momentum of the flow at the impeller exit to equal the average angular momentum in the throat of the volute. The impeller tip velocity, $\mathrm{u}_{2}$, which is used to nondimensionalize the measured velocities is 6.60 $\mathrm{m} / \mathrm{s}$. The dimensionless specific speed, $\omega \mathrm{Q}^{0.50} /(\mathrm{gH})^{0.75}$, of the pump is 0.60 (1583 US units) corresponding to a dimensionless design flow coefficient, $Q /\left(2 \pi \mathrm{r}_{2} \mathrm{bu}_{2}\right)$, of 0.061 . The Reynolds number, $\mathrm{c}_{\mathrm{r} 2} \mathrm{r}_{2} / \nu$, at design conditions is $4.0 \times 10^{4}$, where $c_{r 2}$ is calculated to be the average radial velocity at the impeller discharge calculated from the design flow rate and the discharge area, $\mathrm{Q} /\left(2 \pi \mathrm{r}_{2} \mathrm{~b}\right)$. Figures 1 and 2 depict the impeller and volute geometry. The four bladed impeller, the volute, and splitter all have logarithmic spiral shapes with $16^{\circ}, 83^{\circ}$, and $83^{\circ}$ angles, respectively. Window locations for the laser velocimetry measurements are also shown in Fig. 2. The pump is constructed of Plexiglas and the casing wall is $5.08 \mathrm{~cm}$ thick. Thickness at the windows is reduced to $0.95 \mathrm{~cm}$ to facilitate the use of laser velocimetry.

The flow loop is shown in Fig. 3. This is a closed loop system fed from a 2000 liter reservoir tank. The flow 


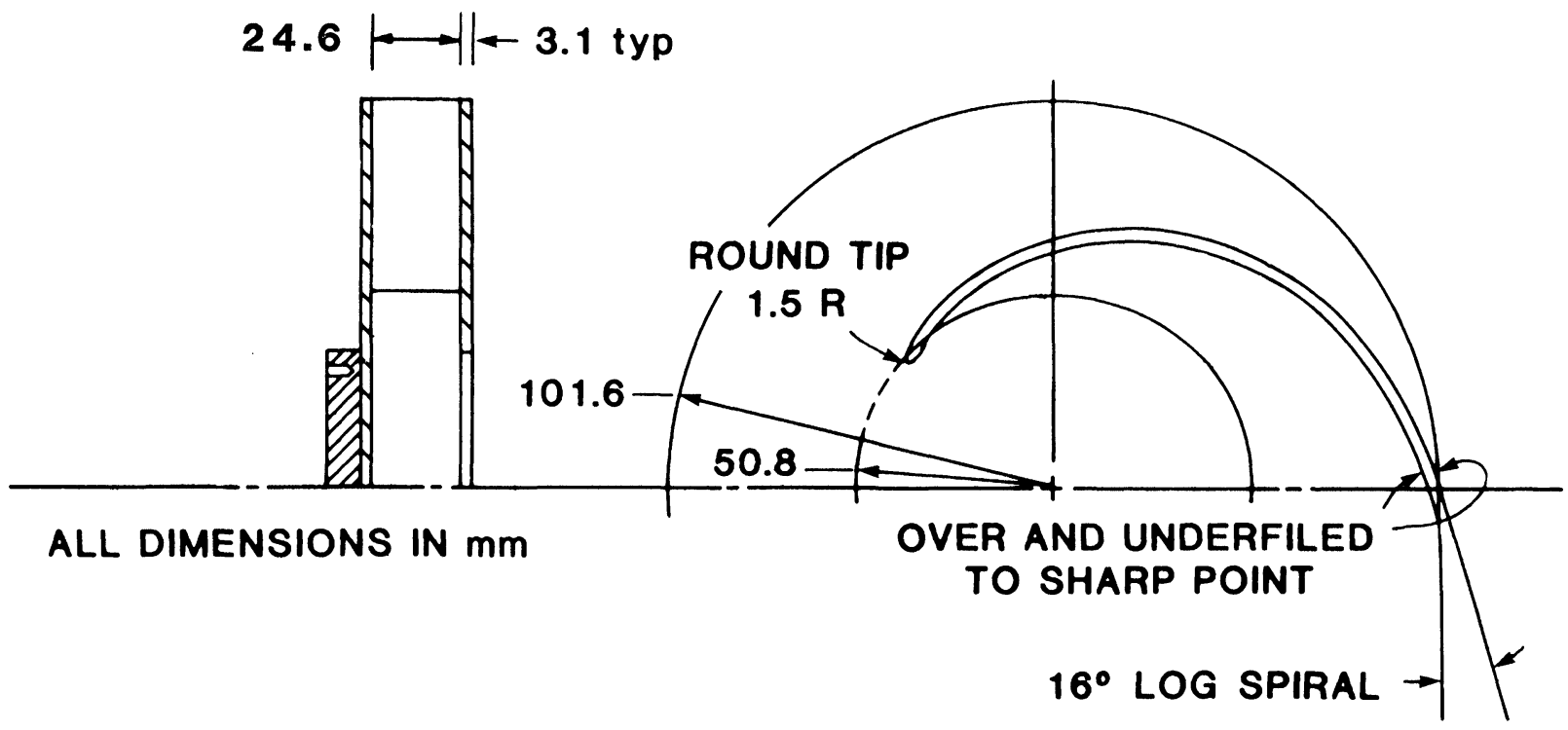

FIGURE 1 Impeller Geometry

straightener in the $76.2 \mathrm{~mm}$ diameter pipe upstream of the pump inlet provides a swirl free inlet flow to the pump. Static pressure taps are located 5 pipe diameters upstream of the impeller inlet and just beyond window 11 in the discharge. Yaw probe traverses in the inlet pipe indicated that the flow was swirl free three diameters upstream at shut off conditions. The second flow straightener, 9 pipe diameters upstream of the orifice meter, conditions the flow for the orifice plate. The globe valve is used to adjust the flow.

The head-capacity curves for the pump assembled with the single volute/single discharge and double symmetric volute/single discharge configurations are shown in Fig. 4. The variation of pump efficiencies are also shown. The efficiency was calculated using Euler's theoretical head assuming no pre-rotation in the fluid, which was documented in earlier tests. These are used to compare the hydraulic operation of the configurations tested, not to document the total efficiency of the pumps. The maximum efficiency in the double volute configuration was $67 \%$ at $3.47 \mathrm{l} / \mathrm{s}$ (55\% of design flow). The single volute configuration exhibited a peak of $69 \%$ at $4.85 \mathrm{l} / \mathrm{s}$ (77\% flow).

\section{Laser Velocimeter}

The laser and optics for this system are shown in Fig. 5. These are mounted on a three directional traveling mechanism which allows easy and accurate positioning of the probe volume in the $\mathrm{x}, \mathrm{y}$, and $\mathrm{z}$ directions. The system is 3-beam frequency shifted, operated in the forward scatter mode. An Argon-ion laser operating at $514.9 \mathrm{~nm}$ and $0.50 \mathrm{~W}$ was the light source. The Bragg cell performs the dual functions of beam separation and frequency shifting.

The frequency of light scattered from the probe volume by naturally occurring particles in the water ( 3 $\mu \mathrm{m}$ diameter) is measured by two $5 / 8$ count burst type signal processors. For coincidence of the two components of velocity, a valid signal was required from both processors within $2 \mu \mathrm{s}$. The impeller angular position is recorded with a gray code shaft encoder which divides each revolution into 256 sectors or "bins". Thus, each blade passage consists of 64 bins. The two velocity signals and the shaft position are recorded onto a dedicated microcomputer. Data rates were typically 50 Hz. "Velocity bias correction" was not required as discussed by Flack (1982) because turbulence intensities were typically below $10 \%$.

\section{Procedure}

Velocities were recorded around the impeller discharge, midway between the hub and shroud, at angles of $0^{\circ}, 45^{\circ}$, $135^{\circ}, 180^{\circ}, 225^{\circ}, 270^{\circ}$, and $315^{\circ}$, corresponding to windows 1, and 3 through 8, respectively, in Fig. 2. Measurements were taken at design flow, and at $80 \%$, $60 \%$ and $40 \%$ of design flow. At window 2 the suction pipe interfered with the collecting optics and no measurements were taken. 


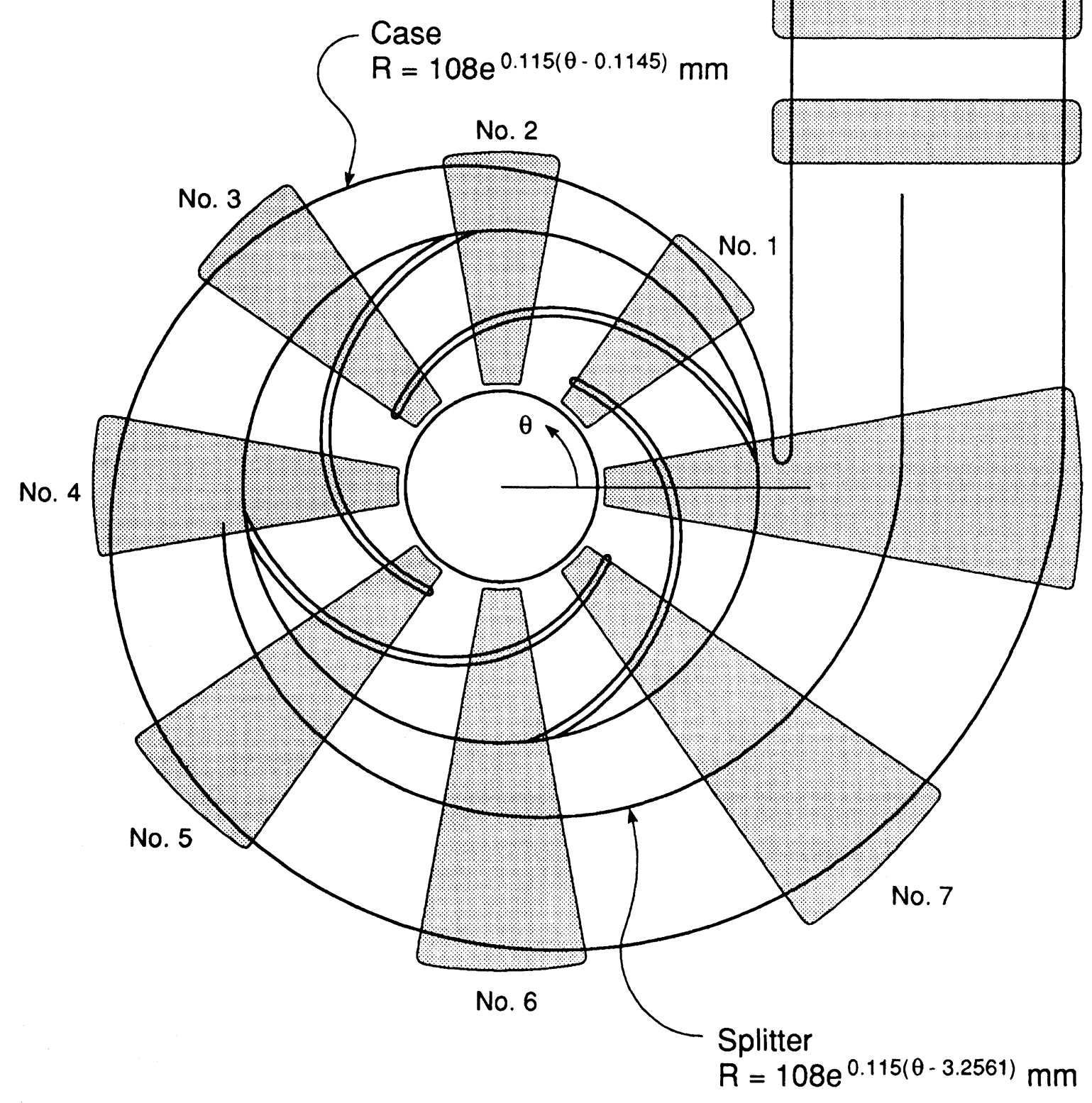

No. 11

No. 10

No. 9

No. 8

FIGURE 2 Volute and Window Locations

A typical test has 2500 data points which corresponds approximately to 10 data points for each of the 256 shaft angle bins. Data recorded in all four blade passages are averaged together to give passage averaged results and 


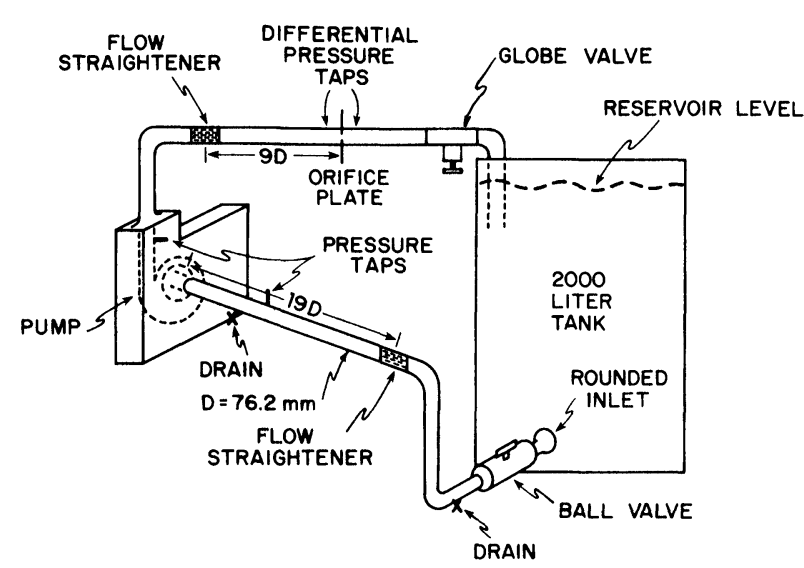

FIGURE 3 Flow Loop

these are presented in this paper. This passage averaging is possible because of the four fold symmetry of the impeller, which results in an average passage with 64 bins and typically 40 samples per bin.

The following section shows measurements of nondimensionalized blade-to-blade velocity profiles, obtained as the blades rotate past the fixed probe volume location. These include radial $\left(\mathrm{c}_{\mathrm{r}}\right)$ and relative tangential $\left(\mathrm{w}_{\theta}\right)$ velocities. The direction of the relative tangential velocity is positive when it follows the curvature of the blade, namely it is positive in the direction opposite of rotation. Figure 6 shows a representative sample of such profiles; these particular data were taken in window 6 at $60 \%$

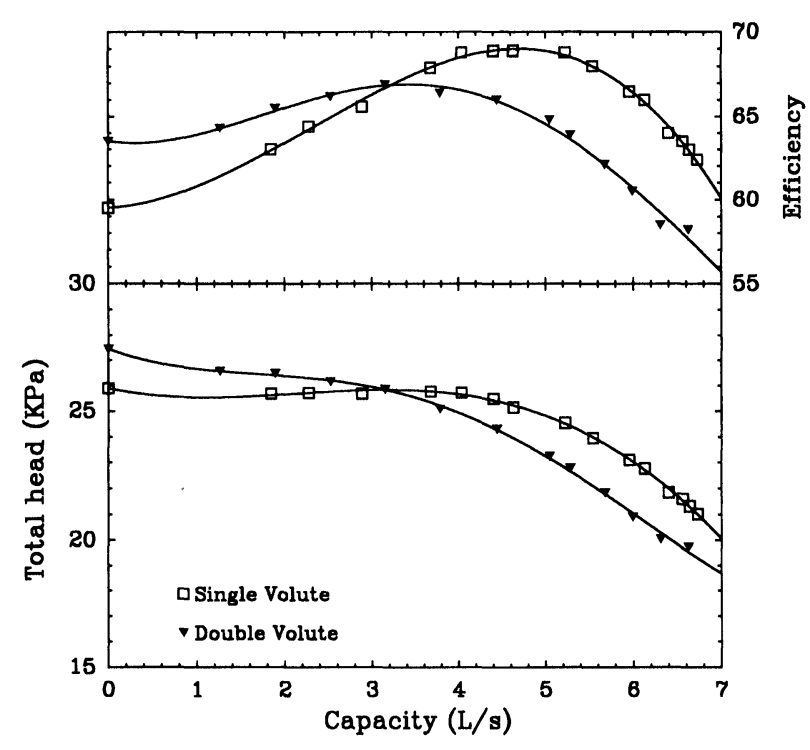

FIGURE 4 Pump Head-Capacity Curve

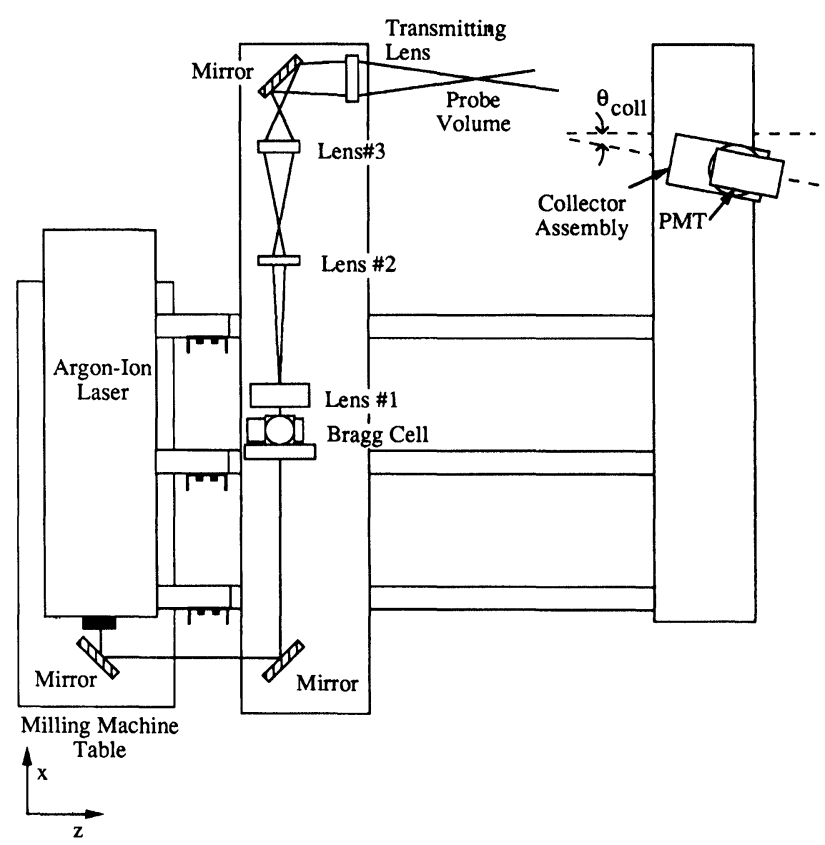

FIGURE 5 Laser Velocimetry Set-up

design flow, The velocities measured at individual bins in the blade passage are depicted as raw data. A fourth order sine series least square fit with nine parameters was then fitted to the raw data. Data for other windows and flow rates showed similar scatter as in Fig. 6. Thus, for the sake of both brevity and clarity, for the results presented in the next section other raw data profiles are not presented; only the least square fits are presented to represent the velocity profiles.

The uncertainty in probe volume position is $0.5 \mathrm{~mm}$ radially, $1 \mathrm{~mm}$ axially, and $0.30^{\circ}$ in the angular position $(\theta)$. The uncertainty in the angular orientation of the probe volume fringes with respect to the reference axes is $0.85^{\circ}$. The latter uncertainty dominates the flow direction uncertainty. Due to processor electronics, the 20:1 odds uncertainty in the nondimensionalized radial velocity component $\left(c_{r} / u_{2}\right)$ is 0.003 and the typical uncertainty in the nondimensionalized tangential velocity component $\left(\mathrm{w}_{\theta} / \mathrm{u}_{2}\right)$ is 0.003 . Flack (1995) has shown that the uncertainty of a curve fit to coherent data points is significantly smaller than that of individual data points because number of data samples is increased. Thus, due to all uncertainties (curve fit, number of samples, beam angles, fringe orientation, and processor) the typical uncertainties in the curve fits to the nondimensionalized radial and tangential velocity components are both 0.009 . These velocity uncertainties do not include the effect of 


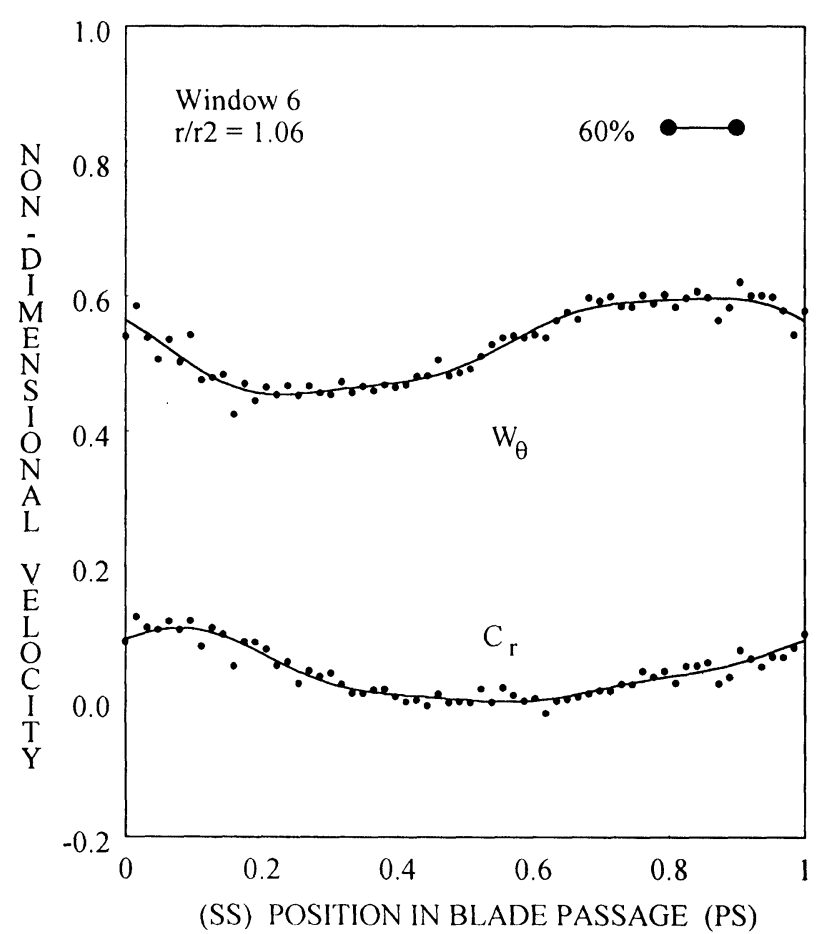

FIGURE 6 Superposition of Measured $c_{r}$ and $w_{\theta}$ Data with Curve Fit Profiles

positional uncertainty in the presence of velocity gradients. The position of the probe volume is uncertain, particularly in the axial direction. Since spatial velocity gradients are present, a misplaced probe volume will measure the incorrect velocity. This spatial uncertainty results in a total curve fit uncertainty of 0.015 for the nondimensionalized radial velocity and 0.015 the nondimensionalized tangential component.

Results presented herein are based on measurements midway between the hub and shroud impeller faces. However, tests were previously documented by Miner $e t$ al. [1989] for the spanwise variation on the velocity profiles. Tests were performed at several positions within the impeller and in the volute. These measurements spanned the distance between the hub and shroud faces. The radial velocity profiles were on occasion nonsymmetrical in the spanwise direction; the tangential components on the other hand were uniform. Near the impeller inlet the radial profiles were skewed towards the hub side due to the $90^{\circ}$ inlet turning of the flow. However, near the impeller exit the flow became much more symmetric and uniform. Namely, for design flow at $\mathrm{r} / \mathrm{r}_{2}=0.62,0.80$ and 0.97 , the maximum variation in $\mathrm{c}_{\mathrm{r}} / \mathrm{u}_{2}$ was $0.20,0.13$, and 0.06 respectively. The varia- tions became negligible for values of $r / r_{2}$ greater than 1.125 .

\section{RESULTS}

\section{Blade-to-Blade Velocity Measurements}

Relative tangential velocity and radial velocity profiles for window 1 at the flow rates tested are shown in Fig. 7. Results are presented at a radius $r / r_{2}=1.06$, representing the impeller discharge. These are suction side (SS) to pressure side (PS) blade-to-blade profiles. The most uniform radial velocity profile was that at design flow. At $80 \%$ of design flow $c_{r}$ decreased from the suction surface to mid-span in the passage and was nearly identical to the profile at the design point in the remaining portion of the passage. For $60 \%$ and $40 \%$ flows, $c_{r}$ was largest in the core region and smallest near the blades; the peak was displaced toward the suction surface for the lower flow rates. Profiles of relative tangential velocity $w_{\theta}$, with exception of $40 \%$ flow, decreased with reduced flow rate, and individual profiles displayed higher velocities toward the blades relative to the core. At $40 \%$ design flow, $\mathrm{w}_{\theta}$ showed a small relative maximum in the core region.

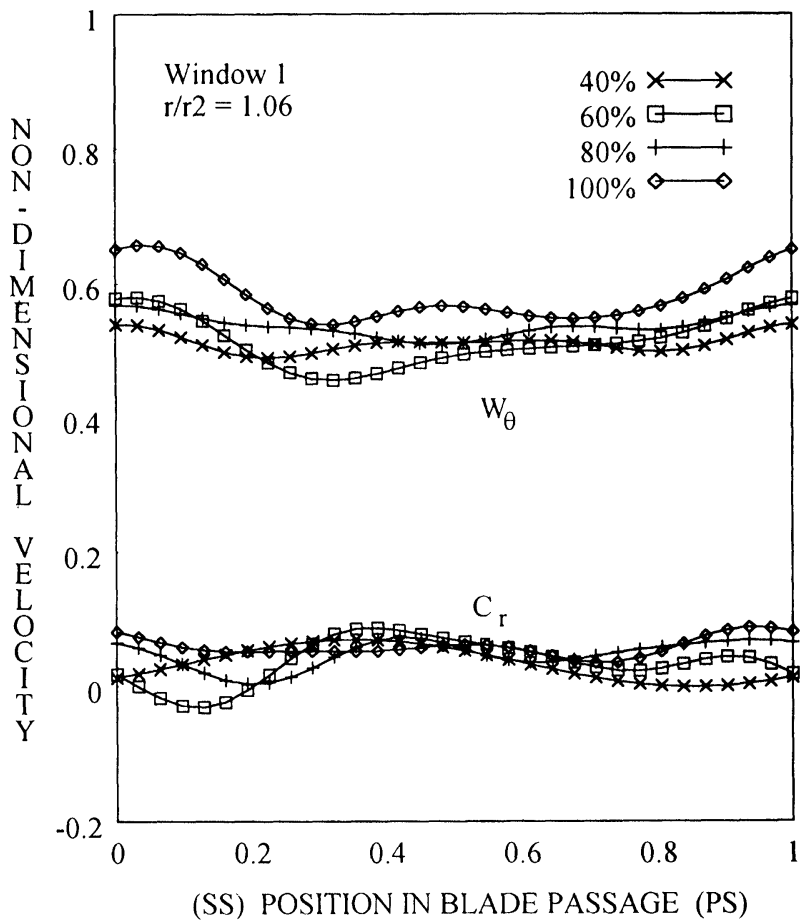

FIGURE 7 Velocity Profiles $w_{\theta}$ and $c_{r}$, Window 1 
Measurements in window 3 exhibited uniformity in all $\mathrm{c}_{\mathrm{r}}$ profiles as illustrated in Fig. 8. The averaged magnitude of these profiles consistently decreased with decreasing flow rates; at design flow, $80 \%$ and $60 \%$ of design flow, profiles were nearly identical from mid-span to pressure surface. Profiles of $\mathrm{w}_{\theta}$ decreased with decreasing flow rate, although toward the blade surfaces, profiles for $40 \%$ flow were higher than the profile at design conditions. In general, as with window 1 , these exhibited higher magnitudes toward the blade surfaces relative to the core passage.

Velocities measured in window 4 show the presence of the cutwater. Namely, profiles of $c_{r}$ (Fig. 9) depict negative (inward) radial flow in the mid-span of the blade passage. At $60 \%$ of design flow, $40 \%$ of the passage experienced recirculation; at $40 \%$ of design flow recirculation was more severe encompassing $80 \%$ of the passage. The cutwater also affected profiles of $\mathrm{w}_{\theta}$. Ideally, if the relative flow angle of the flow remains approximately constant, as the flow rate is decreased, the absolute tangential velocity increases and the relative tangential velocity decreases. Near the cutwater the relative tangential component decreases from $100 \%$ to $80 \%$ capacity (as expected), however, does not decrease further as the flow rate is decreased (Fig. 9). The expected decrease was observed at other windows.

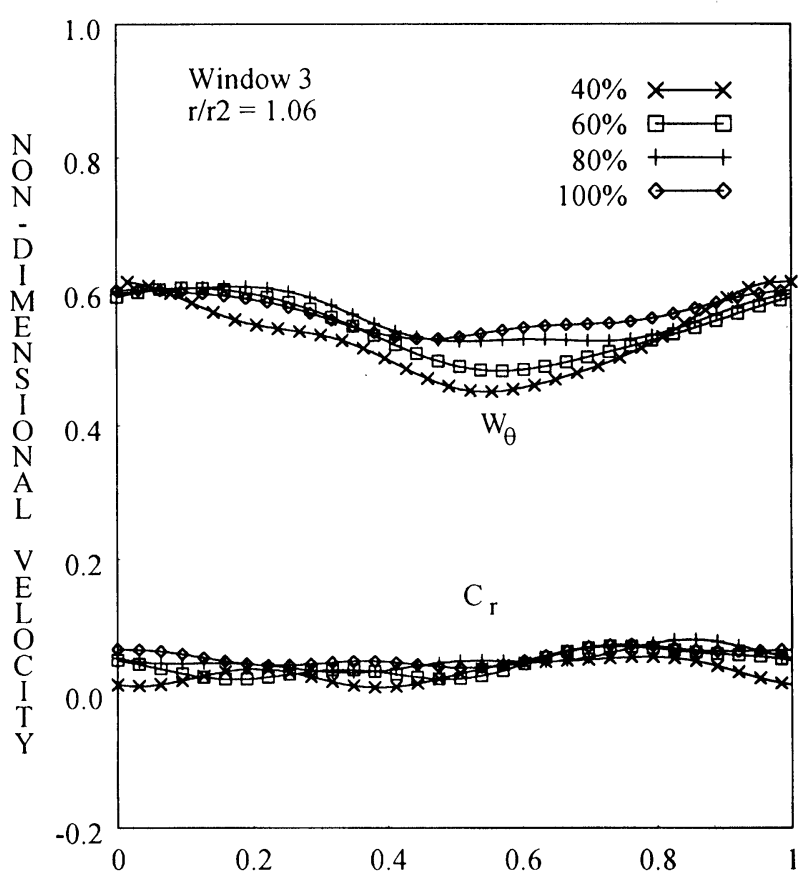

(SS) POSITION IN BLADE PASSAGE (PS)

FIGURE 8 Velocity Profiles $w_{\theta}$ and $c_{r}$, Window 3

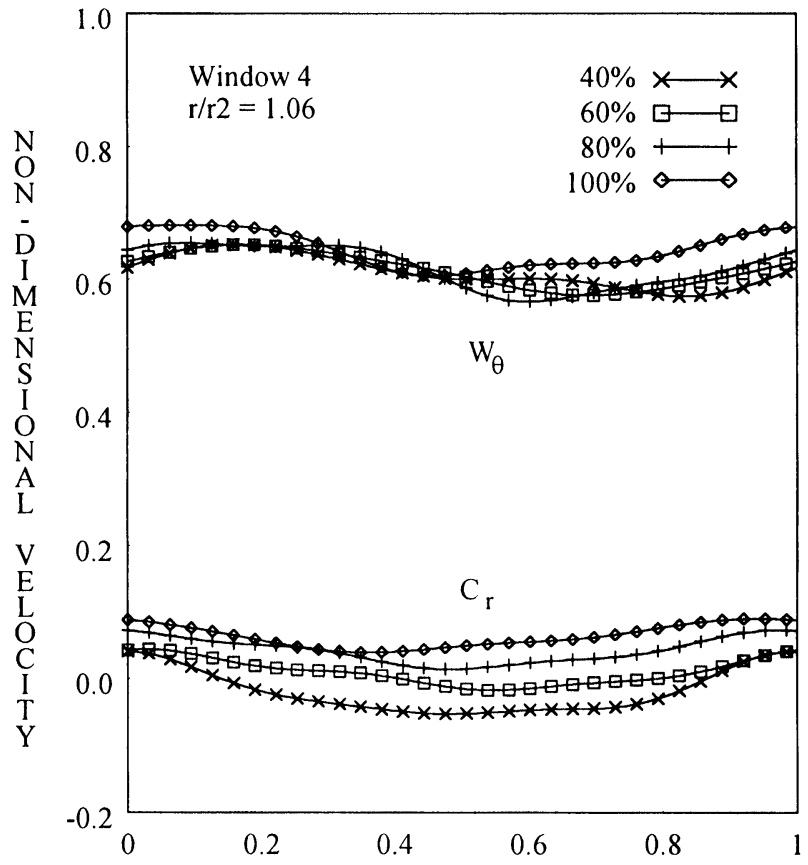

(SS) POSITION IN BLADE PASSAGE (PS)

FIGURE 9 Velocity Profiles $\mathrm{w}_{\theta}$ and $\mathrm{c}_{\mathrm{r}}$, Window 4

Figure 10 shows profiles of $c_{r}$ and $w_{\theta}$ in window 5. The $c_{r}$ profile at design flow exhibited greatest uniformity. Other profiles decreased in magnitude at reduced flow rates. At $80 \%$ of design flow, the decrease was prevalent in the core region; at $60 \%$ of design flow, the decrease expanded to the suction side. At $40 \%$ design flow, $c_{r}$ was highly non-uniform with a peak one-third between the SS and PS and a minimum at the suction surface where a very small recirculation zone is seen. A similar profile was observed in window 1 for $40 \%$ and $60 \%$ of design flow; the two windows are on opposite sides of the volute.

Profiles of $\mathrm{w}_{\theta}$ at design flow, $80 \%$ and $60 \%$ of design flow displayed maximum values toward the blade surfaces and minimums in the core region. The minimum value in the $60 \%$ profile was shifted toward the suction surface identically to its counterpart in window 1 . The $40 \%$ profile showed a minimum toward the suction surface in contrast to the opposing profile in window 1 , where it exhibited a small relative maximum value in the core.

Profiles of $c_{r}$ and $w_{\theta}$ for window 6 are shown in Fig. 11. For $c_{r}$ the uniformity shown at the design point was lost as the flow rate was reduced, due to successive decreases in the core velocity. The loss of uniformity was 


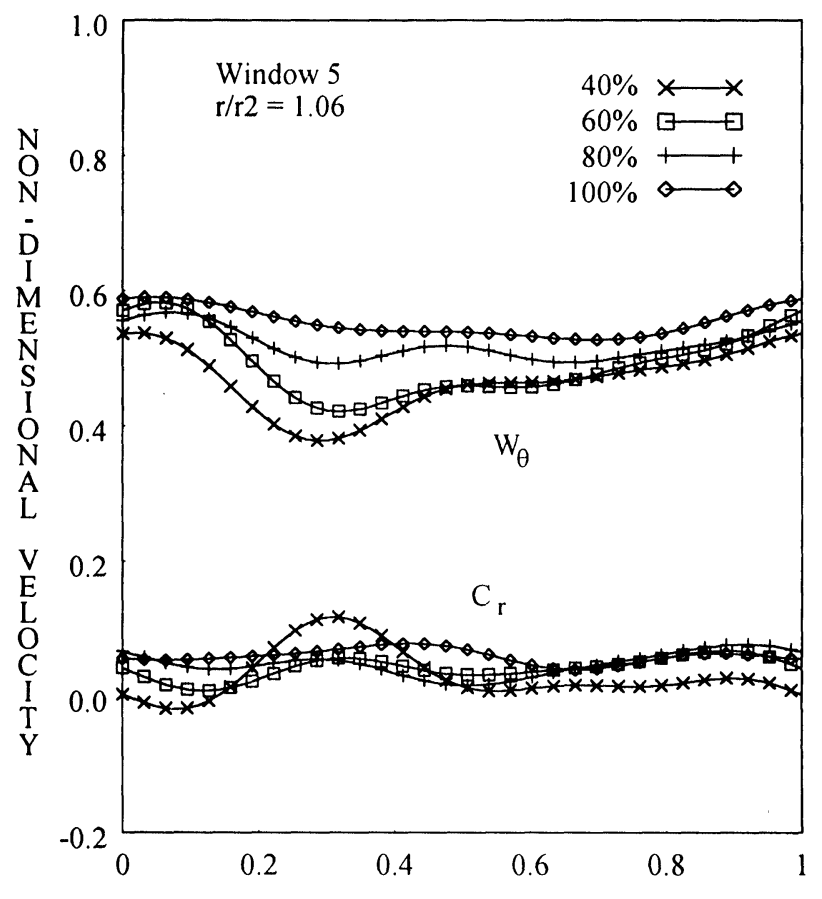

(SS) POSITION IN BLADE PASSAGE (PS)

FIGURE 10 Velocity Profiles $w_{\theta}$ and $c_{r}$, Window 5

accentuated through the rising velocities toward the suction surface. Recirculation was incipient at $60 \%$ of design flow, and occupied a fifth of the passage at $40 \%$ flow. This is the only instance where recirculation was recorded for flows other than in the proximity of the cutwaters. Profiles of $\mathrm{w}_{\theta}$, also illustrated in Fig. 11, showed greater uniformity at and near the design point than for other windows. Profiles consistently showed relative minimums toward the suction surface and relative maximums toward the pressure surface.

Radial velocity profiles measured in window 7 (Fig 12) displayed great uniformity for all flow rates and considerable similarity to those of window 3 , which was located opposite to it. These decreased consistently with reduced flow rates. Again, at $40 \%$ capacity a very small recirculation zone is seen near at the suction surface. Profiles of $\mathrm{w}_{\theta}$ also followed the general trends recorded in window 3 . Velocities were higher near the blades and showed a minimum in the core region. In window 7 the design condition was an exception; it experienced a relative maximum in the mid-section of the passage.

The effects of the tongue on the measurements performed in window 8 were similar to those observed earlier in window 4 . Figure 13 shows the radial velocity profile at both design flow and $80 \%$ flow to be most uniform, although the velocities at $80 \%$ flow were lower than at the design point. A large minimum is shown for lower flow rates; recirculation regions encompass $45 \%$ and $80 \%$ of the blade passage for $60 \%$ and $40 \%$ of design flows, respectively. The $\mathrm{w}_{\theta}$ profiles at $80 \%$ and $60 \%$ of design flow were nearly identical for the entire passage. The profile recorded at $40 \%$ flow was nearly identical to these for the suction surface to mid-span region, but exhibited a lower magnitude from mid-span to the pressure surface. All three profiles were nearly identical throughout the blade passage in window 4 .

The magnitude of the reversed flow at the cutwaters was significant. For example, the maximum non-dimensionalized radial inflow velocity was -0.052 and -0.049 for windows 4 and 8 , respectively; these occurred at $40 \%$ of design flow. Also, the average inflow velocity was found for each window by averaging only the negative radial velocity component. This non-dimensionalized radial inflow velocity was -0.036 and -0.029 for windows 4 and 8 , respectively (again at $40 \%$ flow).

\section{Velocity Trends Compared to Single Volute}

The preceding section documented radial and tangential velocity profiles measured at circumferential positions

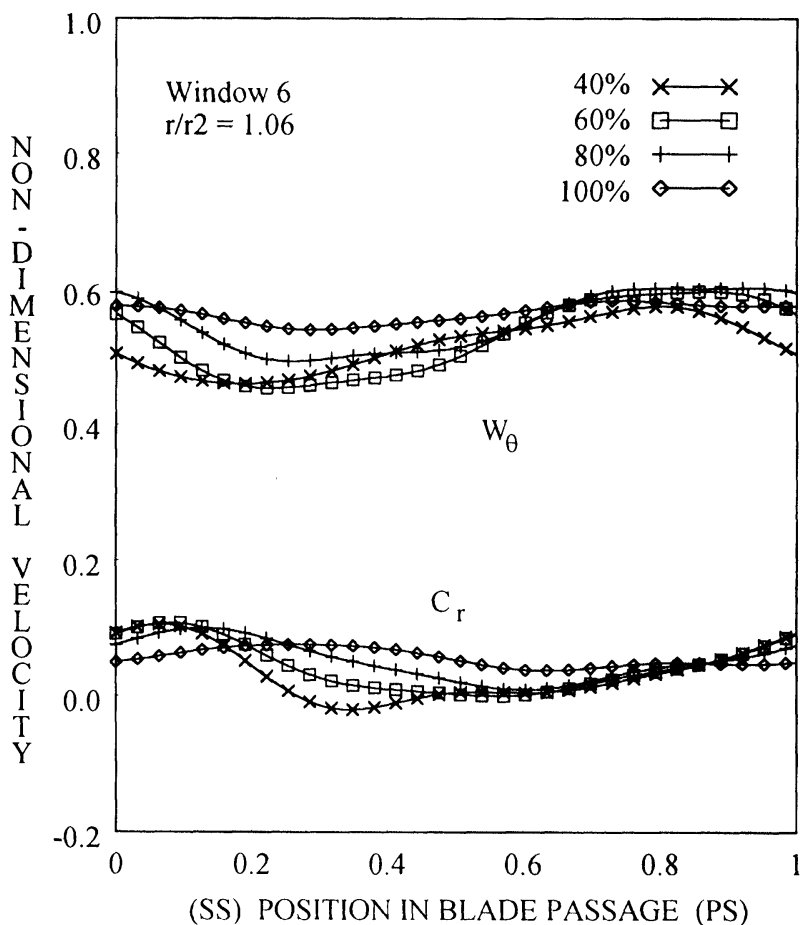

FIGURE 11 Velocity Profiles $w_{\theta}$ and $c_{r}$, Window 6 
around the impeller discharge. Several trends were identified and the significance may be better appreciated when compared to earlier results presented by Miner $e t$ al. (1989) in the same pump assembled as a single volute pump. In this previous study, measurements were made at radial locations of $97 \%$ and $103 \%$ of the impeller exit radius. Over this $6 \%$ range the shape of the blade to blade profiles for a particular window did not change significantly. In addition, changes in the average radial and tangential velocities over this same range wẹre small, and could be attributed to the change in area associated with the change in radius. In general for measurement locations within the radius of the cutwater, significant changes in the blade to blade profiles for a particular window were not present. However, when comparing blade to blade profiles for different windows around the impeller exit, these authors did show the flow to be circumferentially asymmetric even at design flow. Furthermore, for this particular pump the cutwater is $6.3 \%$ greater than the discharge radius. Therefore, measurements at $6 \%$ greater than the discharge radius are still within the radius of the cutwater and should closely represent the velocities at the impeller periphery. For the current geometry, the symmetric volute region increases the symmetry of the flow considerably. The opposing windows showed a commanding similarity for respective

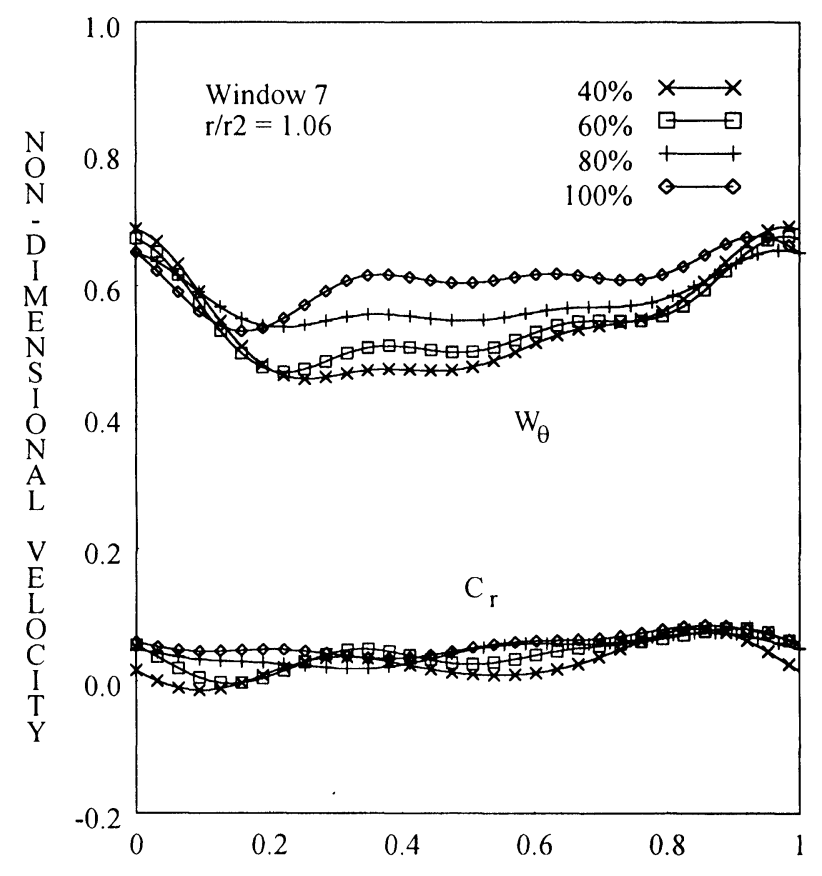

(SS) POSITION IN BLADE PASSAGE (PS)

FIGURE 12 Velocity Profiles $w_{\theta}$ and $c_{r}$, Window 7

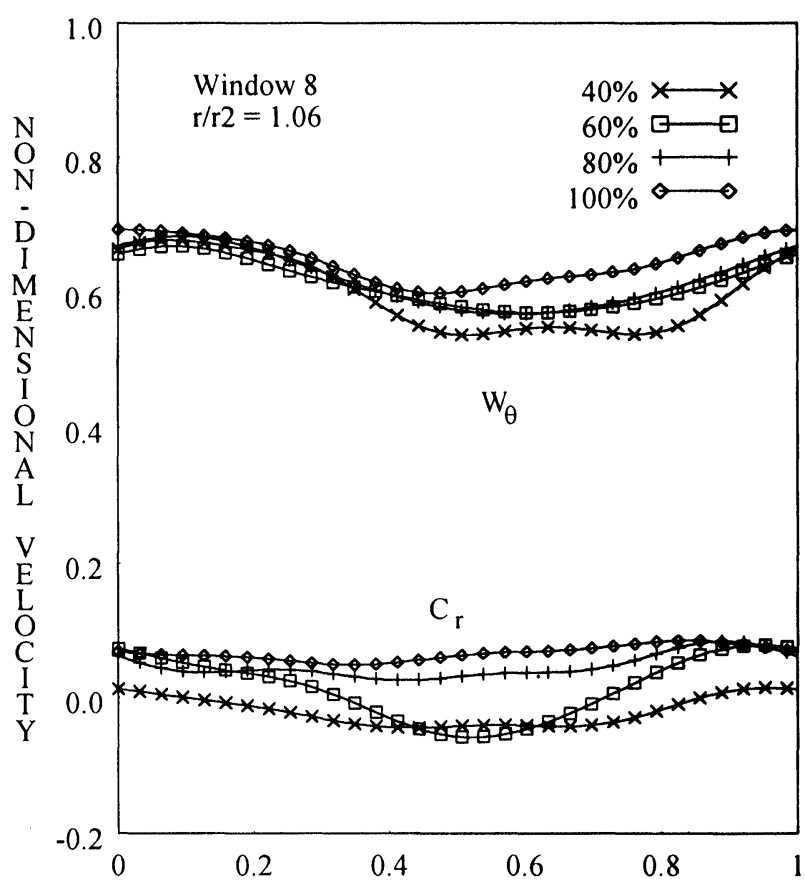

(SS) POSITION IN BLADE PASSAGE (PS)

FIGURE 13 Velocity Profiles $w_{\theta}$ and $c_{r}$, Window 8

operating flow rates; these have been indicated in the preceding section. Furthermore, an increased uniformity in the blade-to-blade profiles was achieved throughout the range of flows tested, as compared to the single volute configuration.

A specific manifestation of this uniformity was appreciated in the reduction of recirculating regions (with exception of the cutwater regions). Significant recirculation was only observed in window 6 mid-way in the passage, and even in this window it was limited to a small region. In this same window, at $40 \%$ of design flow, Miner et al. (1989) measured outward radial flow along the pressure surface and inward radial flow along the suction surface in the impeller. Recirculation at the cutwater was restricted to $60 \%$ and $40 \%$ design flows and took place in the core of blade passage, covering the largest region at the lowest flow rate. Neither cutwater experienced this phenomena at higher flow rates. On the contrary, Miner et al. showed the single volute configuration displayed incipient recirculation for two thirds of the blade passage at the design point.

Here, and in those of Miner et al., profiles for varying operating flow rates displayed greater sensitivity toward the suction side than the pressure side; the profiles exhibited greater disparity from one another near the 
suction surfaces, while they tended to be more identical near the pressure surface.

\section{Time Averaged Impeller Exit Velocities}

Time averaged radial and tangential velocities were calculated by averaging velocity profiles across the blade passage for each window. That is,

$$
\begin{aligned}
& \overline{c_{r}}=\sum_{n=1}^{64} \frac{c_{r_{n}}}{64} \\
& \overline{c_{\theta}}=\sum_{n=1}^{64} \frac{c_{\theta_{n}}}{64} \\
& \overline{w_{\theta}}=\sum_{n=1}^{64} \frac{w_{\theta_{n}}}{64}
\end{aligned}
$$

The total absolute velocity, time averaged over the blade passage, was found from,

$$
\bar{c}=\sqrt{\left(\overline{c_{r}}\right)^{2}+\left(\overline{c_{\theta}}\right)^{2}}
$$

For the velocities measured here, the above time averaged values matched within one percent those otherwise calculated from,

$$
\overline{c^{2}}=\sum_{n=1}^{64} \frac{c_{n_{2}}}{64}=\frac{\sum_{n=1}^{64}\left[c_{r_{n}}{ }^{2}+c_{\theta_{n}}{ }^{2}\right]}{64}
$$

that is,

$$
(\bar{c})^{2} \approx \bar{c}^{2}
$$

Average radial velocities, $\overline{\mathrm{c}}_{\mathrm{r}}$, and total velocities, $\overline{\mathrm{c}}$, are shown in Fig. 14 for the double volute configuration at operating flow rates ranging from design flow to $40 \%$ of design flow. As indicated earlier, velocities were not measured in window 2; however, for further data analysis (calculation of forces, etc.) velocities at $90^{\circ}$ from the tongue, were interpolated from neighboring velocities. The profile at design flow for the single volute configuration is also shown.

In the double volute configuration $\overline{\mathrm{c}}_{\mathrm{r}}$ profiles around the impeller are comprised of two similar segments corresponding to each volute half. The difference at opposing locations is small: at design flow, the maximum difference is $6 \%$ between windows 1 and 5; at $40 \%$ of design flow, the maximum difference is $10 \%$ between these same windows.

The $\bar{c}_{\mathrm{r}}$ velocity profiles at design flow and $80 \%$ of design flow are remarkably uniform around the impeller.

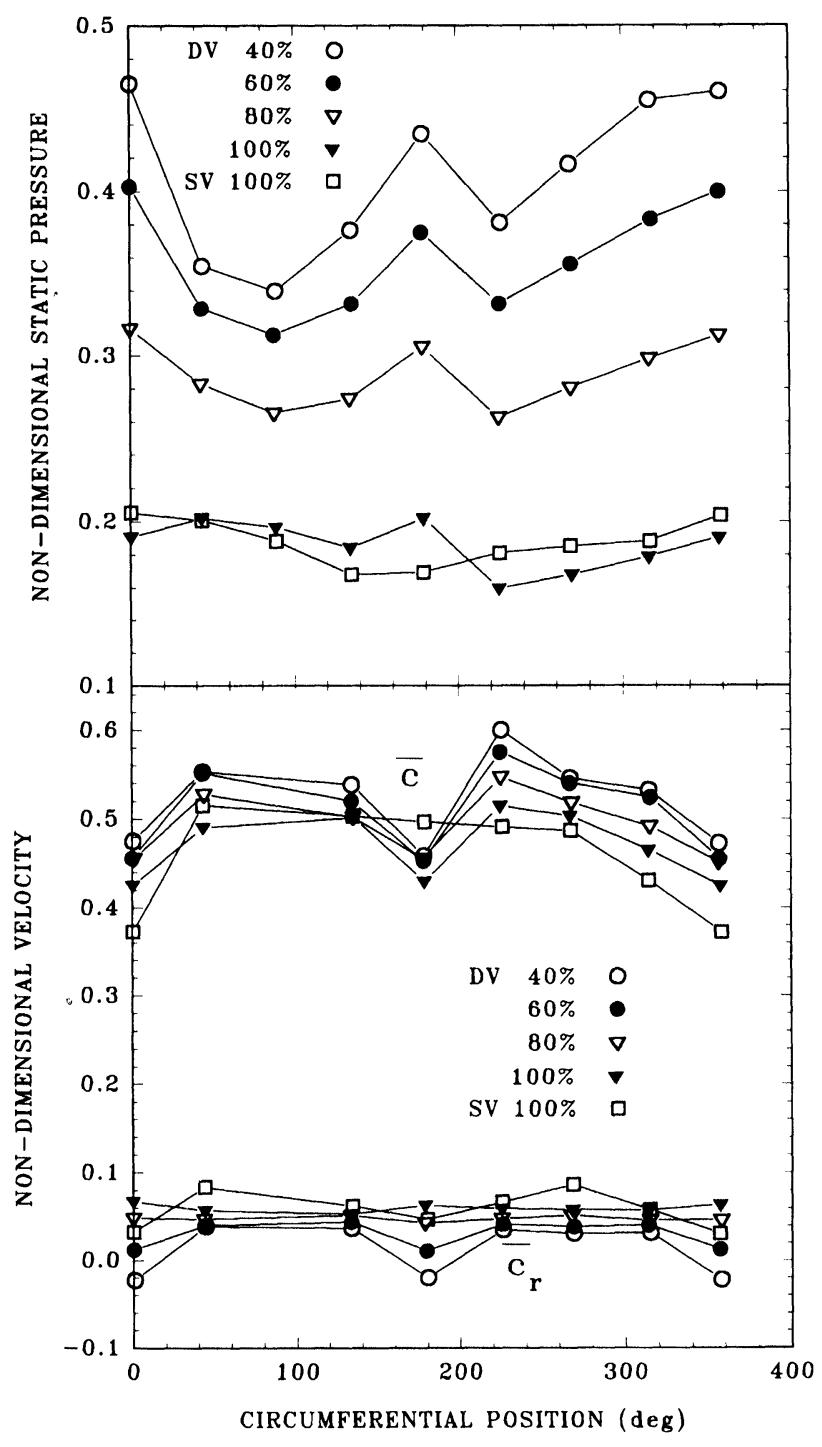

FIGURE 14 Time Averaged $\mathrm{c}_{\mathrm{r}}, \mathrm{c}$ and $\mathrm{p}$ at the Impeller Exit $\left(\mathrm{r} / \mathrm{r}_{2}=\right.$ 1.06)

The maximum deviation from the mean (calculated from the arithmetic mean of the time averaged radial velocities around the impeller) is $13 \%$ and $10 \%$ for design flow and $80 \%$ of design flow, respectively. The deviation for the single volute configuration at design flow was $54 \%$.

Both the $\bar{c}$ and $\bar{c}_{r}$ profiles contribute to the momentum component of force, as well as, to pressure changes, which in turn affect the pressure component of force. The total velocity in the first half $\left(45^{\circ}\right.$ to $\left.180^{\circ}\right)$ decreases slowly towards the splitter cutwater leading edge, at which point it decreases rapidly. After the cut water $\left(180^{\circ}\right.$ to $\left.225^{\circ}\right)$, the velocity rapidly increases, usually more than it decreased across the cutwater. Around the second half $\left(225^{\circ}\right.$ to $\left.360^{\circ}\right)$, the velocity decreases more 
linearly to the primary pump tongue. The single volute geometry at $100 \%$ flow showed a decrease in total velocity only near the tongue. However, the single volute geometry did show a steeper increase than decrease in the direct vicinity of the tongue as also seen in the vicinity of the cutwaters for the double volute configuration.

Non-dimensional static pressures around the impeller discharge are also shown in Fig. 14 for both double volute and single volute configurations. The. opposing trends of pressure from those of velocity would be expected from the Bernoulli model despite its limitations in such a flow. The local static pressure increases preceding the pump tongue and splitter cutwater and decreases behind the obstructions. Figure 14 shows the non-dimensional pressure drop across the splitter cutwater $\left(180^{\circ}\right.$ to $\left.225^{\circ}\right)$ to be 0.042 and independent of flow rate for $60 \%, 80 \%$ and $100 \%$ of design flow. The pressure drop increases to 0.053 for $40 \%$ of design flow. Such consistency is not observed at the primary tongue. Here the pressure drop from $0^{\circ}$ to $45^{\circ}$ increases in an approximately linear sequence: $0.034,0.075$, and 0.113 for $80 \%, 60 \%$ and $40 \%$ of design flow, respectively.

\section{Mass Flow Rate}

The flow rates were also calculated by integrating the time averaged radial velocity profile around the impeller and compared to the directly measured (orifice meter) flow rate. Because of the non-uniformity in the profile near the cutwaters, and because only eight points are present around the impeller, two mass flow distributions were considered. First, the flow was assumed to be uniform over eight equal sized sectors (defined as Distribution 1). Second, experimental investigations, such as those performed by Brownell and Flack (1984) and Miner et al. (1989), are indicative of narrower regions of recirculation in the proximity of the tongue. Because the influence of the cutwaters is localized and less than 1/8 of the exit flow area is affected, each of the sectors near the cutwaters were considered to encompass only $1 / 16$ of the area, which is consistent with the observations of Brownell and Flack and Miner et al., while the adjacent sectors were considered as 5/32 of the area (Distribution 2). The two distributions are depicted in Fig. 15.

The integrated results of the two distributions are shown in Fig. 15. The associated uncertainties inherent in the flowmeter, which increase with reduced flow rates, and in the integration calculation are included in the figures. For the integrated velocity profile the uncertainty is $\pm 0.28 \mathrm{l} / \mathrm{s}$ based on the uncertainty of velocities and the uncertainty that the radial velocity is uniformly distributed over the sectors, are included in the figure. The three

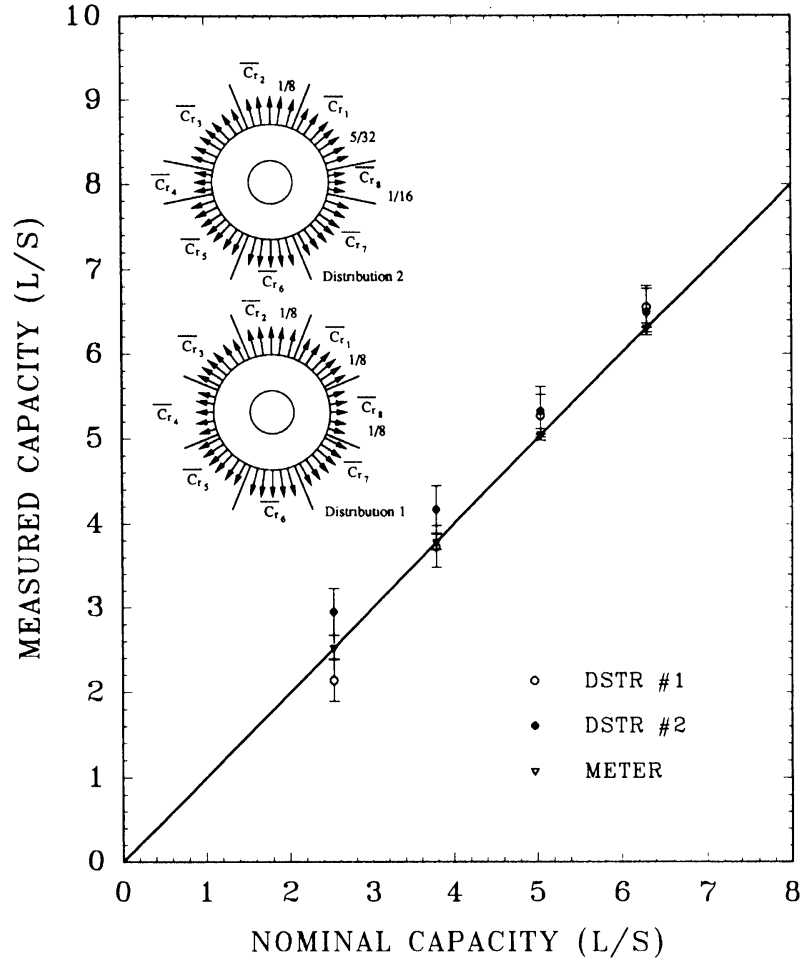

FIGURE 15 Calculated Capacity Based Upon $\mathrm{c}_{\mathrm{r}}$ measurements.

flow rates match within the experimental uncertainty.

\section{Momentum Thrust}

The radial thrust on the impeller due to the non-uniform discharge velocity from the impeller was calculated using the time averaged total and radial velocities shown in Fig. 14. The force vector was calculated from,

$$
\overrightarrow{\mathrm{F}}=\sum_{\mathrm{i}=1}^{8} \dot{\mathrm{m}}_{\mathrm{i}} \overrightarrow{\mathrm{c}_{\mathrm{i}}}=\sum_{\mathrm{i}=1}^{8} \rho \mathrm{A}_{\mathrm{i}} \overrightarrow{\mathrm{c}_{\mathrm{r}_{\mathrm{i}}}} \underset{\mathrm{c}_{\mathrm{i}}}{\overrightarrow{\mathrm{c}_{i}}}
$$

where $\mathrm{i}$ designates windows 1 through $8, \mathrm{~A}_{\mathrm{i}}$ is the area around individual measurement locations upon which the time averaged velocities ( $\overline{\mathrm{c}}$ and $\overline{\mathrm{c}}_{\mathrm{r}}$ ) are considered uniform, and $n_{i}$ is the normal vector to $A_{i}$; for this calculation the sectors are considered to be equal sized. The radial thrust due to non-uniform pressure distribution was measured earlier by de Ojeda et al. (1992). These forces are reproduced in Fig. 16. The non-dimensional magnitude of the pressure forces $\left(\mathrm{F} / 2 \mathrm{Hr}_{2} \mathrm{t}\right)$ are shown for the single volute and double volute configurations at varying flow rates. For reference, the non-dimensional radial thrust from the correlation of Stepanoff (1957) is also presented. 


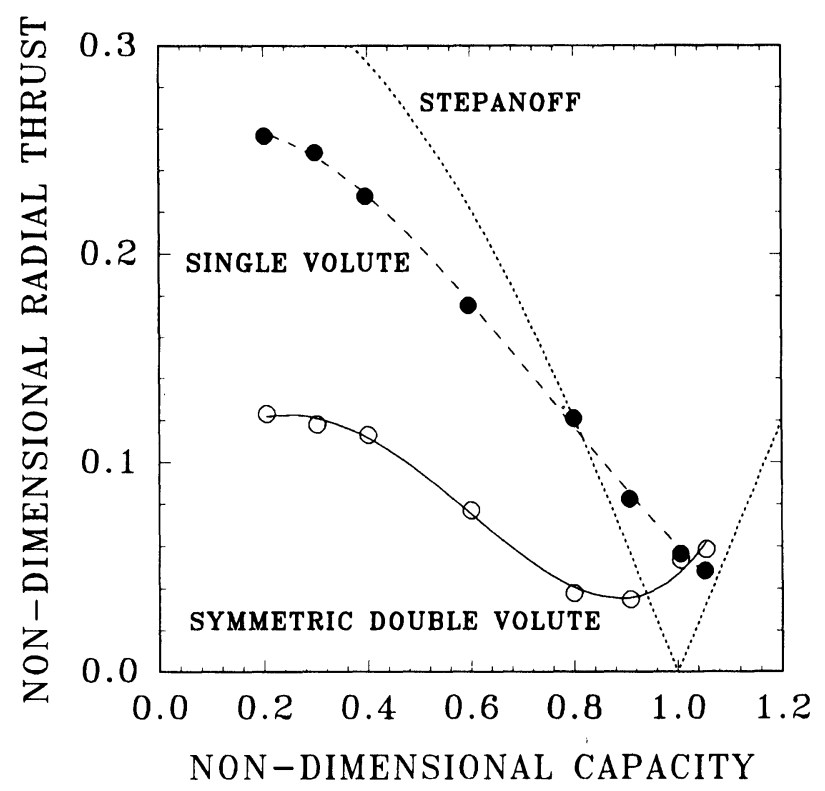

FIGURE 16 Pressure Forces Developed in a Single and Double Volute Pump Configurations

The resulting momentum induced non-dimensional magnitude and orientation of the radial thrust for the double volute configuration are shown in Fig. 17 for flow rates from $40 \%$ to $100 \%$ of design flow. Also shown are the magnitude and orientation relative to the horizontal direction (Fig. 2) of the total force obtained by vectorially combining the pressure and momentum contributions. This figure also depicts the momentum induced radial thrust developed by the single volute configurations calculated based on data from Miner et al. (1989) for the design point.

The contribution of the DV nondimensional momentum force is maximum at design flow $(0.0251)$ and at an orientation of $175^{\circ}$. For this flow rate the nondimensional total force is 0.0624 at $232^{\circ}$. For flow rates below the design point the momentum contribution decreases. At $40 \%$ of design flow, the nondimensional momentum force is calculated to be 0.0018 at $183^{\circ}$, and is considered negligible compared with the nondimensional total force of 0.1112 at $113^{\circ}$. The nondimensional momentum force calculated in the single volute configuration at design flow is 0.023 at $162^{\circ}$, which is very similar in magnitude and direction to that for the double volute pump.

Even though the momentum flux contribution to the force is greatest at the design condition, because of the pressure induced contribution, the overall largest forces are developed at the lower flow rates. For example, at $60 \%$ design flow and lower, the pressure forces are largest, whereas the momentum forces become negli- gible. Fig. 16 shows the effectiveness of the double volute in reducing the radial thrust due to non-uniform pressure distributions. At $20 \%$ design flow the double volute exhibits over $50 \%$ reduction in radial thrust over the single volute pump configuration.

\section{Losses in the Volute}

Local head at a particular location around the impeller exit is found from pressure and velocity measurements at different locations around the impeller exit. The average head around the impeller is calculated from the sum of local heads weighted by their respective time averaged radial velocity and area. Total developed head on the contrary is calculated from pressure and velocity measurements at the discharge flange. By comparing the average of the local head around the impeller to the total

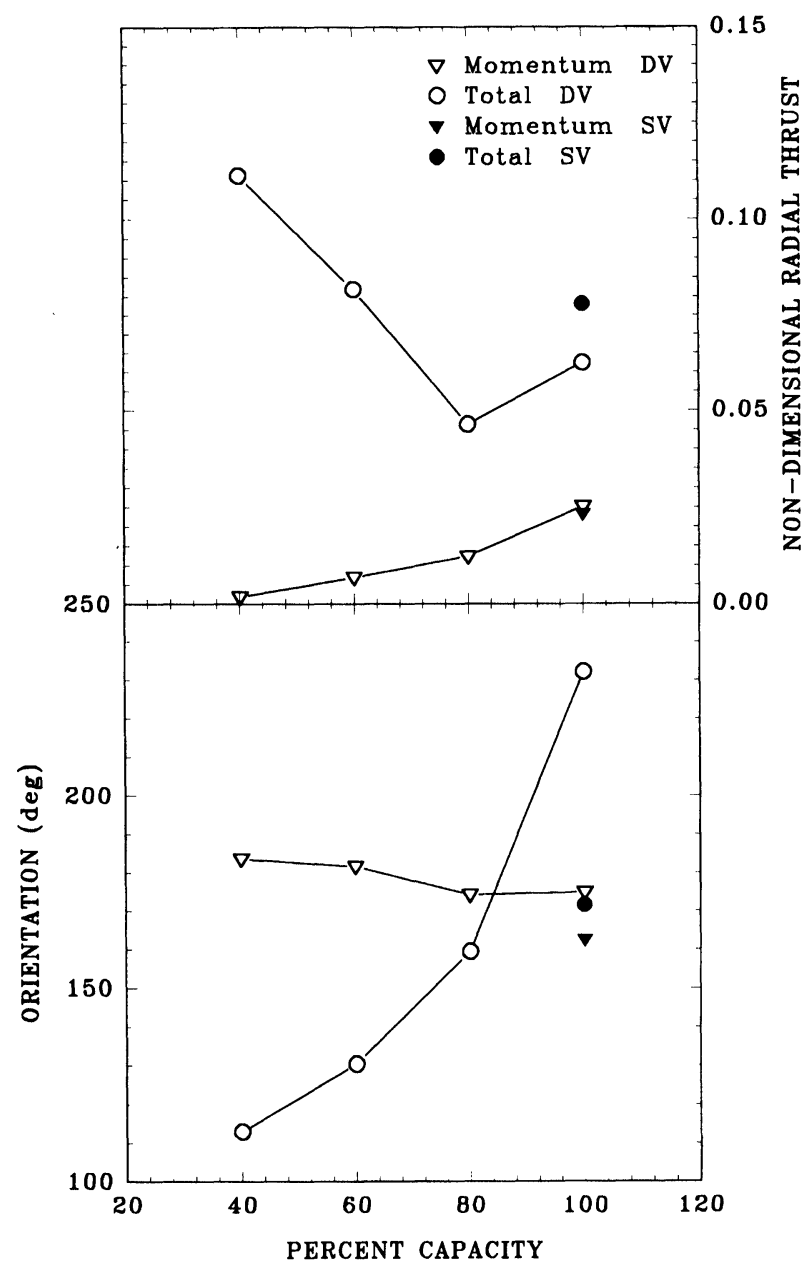

FIGURE 17 Magnitude and Orientation of the Momentum and Total Thrust 
developed head at the discharge flange one can calculate the losses in the volute originating from mixing and friction. Namely,

$$
\Delta \mathrm{H}_{\text {loss }}=\frac{\sum_{\mathrm{i}=1}^{8} \dot{\mathrm{m}}_{\mathrm{i}} \mathrm{H}_{\mathrm{i}}}{\dot{\mathrm{m}}_{\mathrm{D}}}-\mathrm{H}_{\mathrm{D}}=\sum_{\mathrm{i}=1}^{8}\left[\frac{\mathrm{A}_{i}}{\mathrm{~V}_{\mathrm{D}} \mathrm{A}_{\mathrm{D}}} \overline{\mathrm{c}}_{\mathrm{r}_{\mathrm{i}}}\right] \mathrm{H}_{\mathrm{i}}-\mathrm{H}_{\mathrm{D}}
$$

Due to the losses in the volute the total developed head will be lower than the average of the local head at the discharge. The two mass flow distributions discussed earlier were considered to find the average head at the impeller exit. Average local and total developed head in the double volute are shown in Fig. 18.

The first distribution (equally divided sectors) fails to represent adequately the losses in the volute for low flow rates; for flow rates below $80 \%$ design flow the loss is negative, a physically unattainable phenomena. The second distribution shows a realistic scenario with increasing losses for reduced flow rates. The loss based on the second distribution is consistent with the earlier discussion toward maintaining that the area of recirculation is limited to the vicinity of the tongues. Both distributions indicate realistic losses for the design condition. Also, both distributions indicate minimal volute losses for $80 \%$ design flow. For both $80 \%$ and $100 \%$ design flow the mass flow rates agreed within $1 \%$ for the two distributions. For lower flow rates, since the two distributions lead to significantly different mass flow rates and losses, one must conclude that more details of the flow are needed near the cutwaters before dependable volute loss models can be presented. Although four of the data points in Fig. 18 are considered undependable and indicated as so, they are included in this paper to document the results for the two distributions and to indicate the sensitivity to the chosen distributions.

The losses represented in Fig. 18 for the double volute (mass flow distribution \#2) and single volute have been quantified in Table 1. The percentage losses have been calculated for a given capacity by dividing the difference between the corresponding total head and averaged head around the impeller by the averaged head around the impeller. The causes and relative importance of these losses are considered in the Discussion section.

\section{Slip Factor}

The slip factor for the double volute configuration was calculated for flow rates ranging from $40 \%$ of design flow to design flow at the impeller discharge $\left(r / r_{2}=1.06\right)$. The theoretical absolute tangential component of veloc-

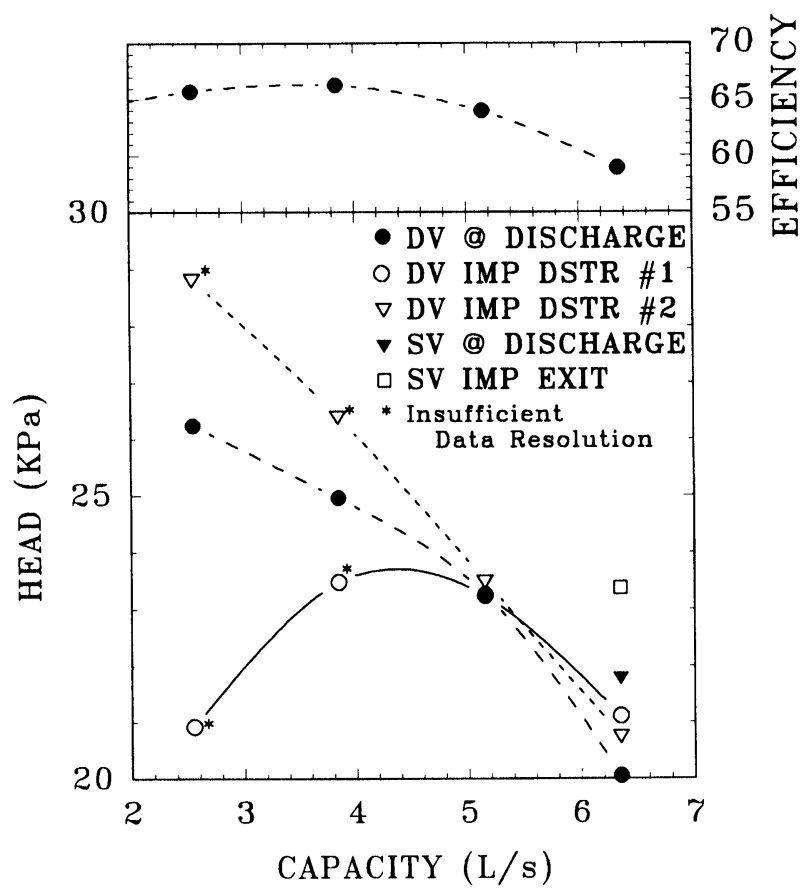

FIGURE 18 Local and Total Developed Head

ity is calculated from the time averaged radial measurements of velocity across the blade passage similar to that done by Miner et al. (1989) from Busemann (1928):

$$
\mathrm{h}_{0}=\left[1-\frac{\mathrm{zt}_{\mathrm{b}}}{2 \pi \mathrm{r} \sin \beta}\right]^{-1}\left[\frac{\mathrm{Q}}{2 \pi \mathrm{rbu}}\right] \cot \beta+\frac{\overline{\mathrm{c}}_{\theta}}{\mathrm{u}}
$$

The values of slip factor are shown in Fig. 19 for windows 1 through 4 , and 5 through 8 , corresponding to first and second volute halves. They are relatively small because of the strong influence of the volute upon the flow at the impeller discharge. For the design condition the double volute configuration exhibits slip factors ranging approximately from 0.64 to 0.72 around the impeller. The values of slip factor are relatively constant with varying flow rates at any window location around the impeller discharge with exception of windows 4 and 8. The severe recirculation at these locations causes a drop in slip factor at low flow rates.

Figure 19 also displays a general trend of decreasing slip factor when traversing from one cutwater to the next, the decrease being more pronounced when reaching the cutwater, particularly at lower flow rates. This is also seen in Fig. 20, where slip factor values calculated from data by Miner et al. (1989) for the single volute pump are also shown. In general the values of slip factor are higher for the single volute except at the cutwater located in 


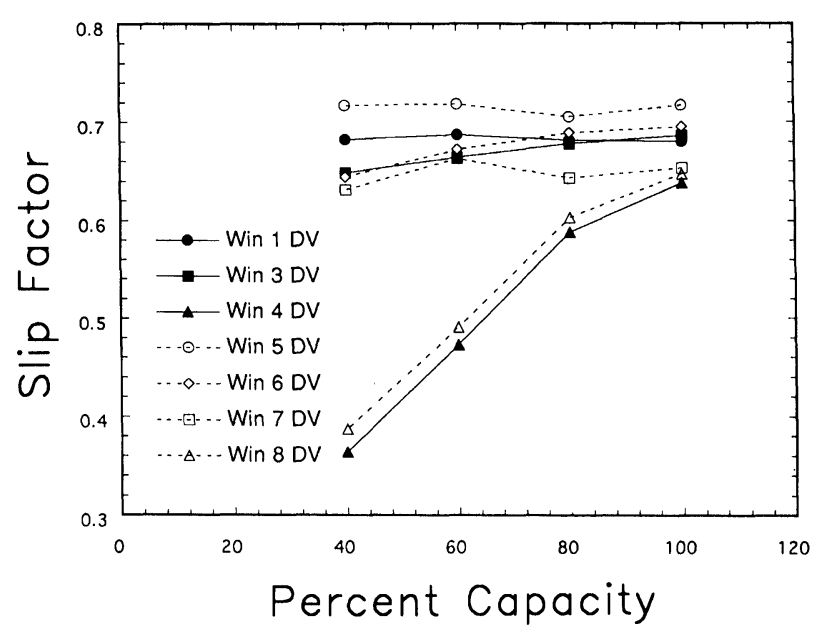

FIGURE 19 Local Slip Factor for Double Volute Pump

window 8 . For the double volute and single volute the average slip factors were 0.67 and 0.68 , respectively. Values for the slip factor based on the research of Busemann (1928) and Wiesner (1967) for this pump geometry are 0.76 and 0.80 , respectively. Miner et al. (1989) calculated the slip factor for the single volute geometry using an average radial velocity, $\mathrm{c}_{\mathrm{r}}$ (averaged circumferentially and axially), in place of $\mathrm{Q} / 2 \pi \mathrm{rb}$. The value of slip factor using this method was within $5 \%$ of the value reported in this paper. Miner et al. also reported axial variations in $\mathrm{w}_{\theta}$ of less than $5 \%$ and axial variations of $c_{\theta}$ less than $2 \%$; thus, the axial variation in velocity does not account for the current deviations from the predictions of Busemann and Wiesner.

\section{Discussion}

The losses incurred in overall pump performance by introducing a splitter in the discharge channel are apparent when considering the increased loss of efficiency as compared to the single volute pump (Fig. 4). However, the head-capacity curve showed that additional losses were not manifested throughout the range of operation but only for flows over 55\% of design flow. For flow

TABLE 1

Volute Losses in Two Pump Configurations

\begin{tabular}{llllll}
\hline & DV-100\% & DV-80\% & DV-60\% & DV-40\% & SV-100\% \\
\hline $\begin{array}{l}\text { Losses (KPa) } \\
\begin{array}{l}\text { Percent losses } \\
\text { based on }\end{array}\end{array}$ & 0.62 & 0.28 & 1.22 & 2.69 & 1.67 \\
discharge & $2.98 \%$ & $1.21 \%$ & $4.65 \%$ & $10.22 \%$ & $7.14 \%$ \\
\hline
\end{tabular}

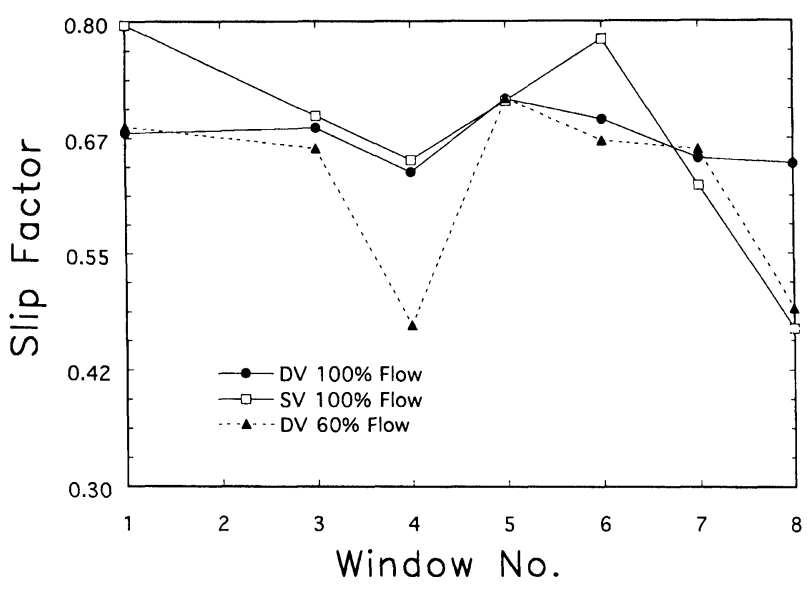

FIGURE 20 Local Slip Factor at Selected Flows for Double and Single Volute Pumps

rates larger than $80 \%$ of design flow, the decrease in total head averaged $8 \%$; for flow rates below $40 \%$ of design flow, the increase performance averaged 3\%. The reason for this phenomena may be understood in light of two contributing factors to efficiency.

Friction contributes to an increased loss of performance in the double volute configuration rising from the increased boundary layer area introduced by the splitter. Friction losses predominate at high flows, near the design point, where other losses, such as those caused by mixing are considered small given the favorable matching of the flow at the impeller exit with the volute flow. This is supported by the uniform velocity profiles near the design point.

In the case of a pump, velocities in the discharge channel decrease with lower capacities. In this region friction decreases with reduced velocities. Because of the impelling action of the blades, fluid will always be transported to and from the impeller. Friction losses will always be present and although they will decrease with decreased flow rates, for the purpose of this discussion, they are considered constant.

Mixing losses account for the remaining contribution toward loss of efficiency. These losses predominate at low flows were the matching of impeller and volute flows is weakened. If one considers friction losses to remain constant, Fig. 18 shows mixing losses increase rapidly for reduced flow rates.

The representation of the average head at the impeller exit and total head curves yield therefore the following qualities: (a) The reduced magnitude of the total head curve for the double volute configuration for high flows (over 55\% of design flow) is a result of additional friction from the boundary layers; (b) The increased 
magnitude of the total head curve for the double volute configuration for low flows (under $55 \%$ of design flow) is a result of better control over recirculation.

Evidence toward the reduction of recirculation regions was demonstrated; recirculation was documented as restricted to the tongue areas for $60 \%$ design flow and lower in the double volute configuration. On the contrary, measurements by Miner et al. (1989) showed incipient recirculation in the single volute at design flow in the proximity of the tongue, and extensive recirculation for $40 \%$ design flow at window 6 .

The double volute pump configuration therefore exhibits an increase and decrease of hydraulic efficiency for low and high ranges of flow, respectively, when compared to the single volute configuration. Most significantly, however, this quality is accompanied by important reductions of radial thrust, ranging from 50 to 70 percent as documented in the results presented here.

\section{CONCLUSIONS}

In this paper velocity data obtained with laser velocimetry is presented for a double volute centrifugal pump. The velocity data complements previously obtained pressure data for the same pump. The velocity data also complements previously obtained velocity data for a single volute version of the same pump. The differences of the internal flow fields between the two pump types were determined. Also, the data was used to evaluate the momentum induced radial thrust; volute losses, and slip factor. Specific conclusions are:

1. Both the time averaged velocities and the blade-toblade velocity profiles were symmetric around the impeller periphery. Such symmetry was not encountered with the pump assembled as a single volute pump. Previous pressure measurements around the impeller also demonstrated improved symmetry as compared to a single volute pump.

2. The double volute also yielded increased uniformity in individual blade-to-blade profiles. A specific manifestation of the increased uniformity is shown by reduced recirculation. Recirculation was restricted to regions in the vicinity of the cutwaters for flow rates $60 \%$ of design flow and lower. For the single volute pump the recirculation also occurred at higher flow rates and was of greater magnitude.

3. Velocities averaged in time across the blade passage were used to evaluate the contribution by the momentum fluxes to the radial thrust on the impeller. These forces were found to be most significant near the design point and equal to $40 \%$ of the pressure induced component. At $40 \%$ of design flow the momentum contribution was negligible.

4. At low flow rates the pressure induced radial force for the double volute configuration was approximately $50 \%$ of the force for the single volute pump.

5. Efficiency of the double volute pump, when compared to the single volute configuration, showed increased efficiency for flow rates approximately below $55 \%$ of design flow, and decreased efficiency at higher flow rates. At high flow rates the decreased performance of the double volutes was attributed to increased boundary layer friction. At lower flow rates the increased performance was attributed to better control of the recirculation regions.

6. Volute losses are seen to increase by a factor of 3 as the flow rate is decreased from $100 \%$ to $40 \%$ flow for the double volute pump. The volute loss at design flow is about $40 \%$ of the single volute loss.

7. The local slip factor was seen to be symmetric around the impeller periphery. The average slip factor was shown to be approximately the same for the two volute configurations at the design flow rate.

$\begin{array}{ll}\text { Nomenclature } & \\ \mathrm{A} & \text { flow area } \\ \mathrm{b} & \text { impeller flow passage width } \\ \mathrm{c} & \text { absolute velocity } \\ \mathrm{D} & \text { impeller diameter } \\ \mathrm{F} & \text { force } \\ \mathrm{H} & \text { head } \\ \mathrm{h}_{\mathrm{o}} & \text { slip factor } \\ \mathrm{n} & \text { normal vector } \\ \mathrm{Q} & \text { capacity } \\ \mathrm{r} & \text { radius } \\ \mathrm{t} & \text { impeller width including shrouds } \\ \mathrm{t}_{\mathrm{b}} & \text { blade thickness } \\ \mathrm{u} & \text { impeller tangential velocity } \\ \mathrm{w} & \text { relative velocity } \\ \mathrm{z} & \text { number of blades } \\ \beta & \text { blade angle } \\ \nu & \text { kinematic viscosity } \\ \rho & \text { density } \\ \omega & \text { angular speed } \\ & \end{array}$

$\begin{array}{ll}\text { Subscripts } & \\ 2 & \text { impeller outer radius } \\ \text { D } & \text { discharge } \\ \text { i } & \text { window number } \\ \text { n } & \text { bin position } \\ \text { r } & \text { radial component of velocity } \\ \theta & \text { tangential component of velocity }\end{array}$

Acknowledgement

This research was sponsored by the Rotating Machinery and Controls (ROMAC) Industrial Research Program at the University of Virginia. 


\section{References}

Adkins, D.E. and Brennen, C.E., 1988, "Analysis of Hydrodynamic Radial Forces on Centrifugal Pump Impellers," Trans. ASME, Journal of Fluids Engineering, Vol 110, No. 1, pp 20-28.

Agostinelli, A., Nobles, D., and Mockridge, C.R., 1960, “An Experimental Investigation of Radial Thrust in Centrifugal Pumps," Journal of Engineering for Power, Trans. ASME, Vol. 82, No. 2, pp. 120-126.

Beaudoin, R. J., Miner, S. M., and Flack, R. D., 1992, "Laser Velocimeter Measurements in a Centrifugal Pump with an Orbiting Impeller, ASME Transactions, Journal of Turbomachinery, Vol. 114, No. 2, pp. 340-349.

Brownell, R.B, and Flack, R.D., 1984, "Flow Characteristics in the Volute and Tongue Region of a Centrifugal Pump," Paper No. 84-GT-82, ASME International Gas Turbine Conference, The Netherlands.

Busemann, A., 1928, "Das Folderhohen verhaltnis radialer Kreiselpumpen mit Logarithmischapiraligen Schaufeln," Zeitschrift für Angewandte Mathematik und Mechanik, Vol. 8, No. 5, pp. 372-384.

de Ojeda, W., Flack, R.D., and Miner, S.M., 1992, "Pressure Distributions in a Single and Two Versions of a Double Volute of a Centrifugal Pump," Paper 92-GT-20, ASME International Gas Turbine Conference, Cologne, Germany.

Flack, R. D., 1982, "Influence of Turbulence Scale and Structure on Individual Realization Laser Velocimeter Biases," Journal of Physics E - Scientific Instruments, Vol. 15, No. 10, pp. 1038-1044.

Flack, R. D., 1995, "Uncertainty of a Least Squares Curve Fit Through Points with Known Uncertainties," International Journal of Mechanical Engineering Education, accepted for publication.

Guinzburg, A., Brennen, C.E., Acosta, A.J., and Caughey, T.K., 1992, "The Effect of Inlet Swirl on the Rotordynamic Shroud Forces in a
Centrifugal Pump," Paper 92-GT-126, ASME International Gas Turbine Conference, Cologne, Germany.

Hamkins, C.P., and Flack, R.D., 1987, "Laser Velocimetry Measurements in Shrouded and Unshrouded Radial Flow Pump Impellers," Journal of Turbomachinery, Trans. ASME, Vol. 109, No. 1, pp. 70-78.

Howard, J. H. G., Abramian, M., and Herman, P., 1987, "Experimental Investigation of Impeller and Volute Flow Fields for a Low Specific Speed Pump with Single and Double Volutes," Proceedings of the ASME/JSME Thermal Engineering Joint Conference, ASME I0219B, pp. 51-61.

Jery, B., Brennen, C.E., Caughey, T.E., and Acosta, A.J., 1985, "Forces on Centrifugal Pump Impellers," Proceedings of the Second International Pump Symposium, Texas A \& M University, College Station, TX, pp. 21-29.

Kanki, H., Kawata, Y., and Kawantani, T., 1981, "Experimental Research on the Hydraulic Excitation Force on the Pump Shaft," ASME Paper No. 81-DET-71.

Miner, S.M., Beaudoin, R.J., Flack, R.D., 1989 "Laser Velocimetry Measurements in a Centrifugal Flow Pump," Trans. ASME, Journal of Turbomachinery, Vol. 111, No. 3, pp. 205-212.

Ohashi, H., Shoji, H., Yanagisawa, S., and Tomita, K., 1981, Experimental Study of Fluid Forces on Whirling Centrifugal Impeller, in Vaneless Diffuser," ASME Winter Annual Meeting, Fluid Structures Interactions in Turbomachinery, pp 57-62.

Stepanoff, A.J., 1957, Centrifugal and Axial Flow Pumps - Theory, Design, and Application, 2nd ed., Wiley.

Wiesner, F.J., 1967, "A Review of Slip Factors for Centrifugal Impellers," Trans. ASME, Journal of Engineering for Power, Vol. 89, pp. 558-572. 

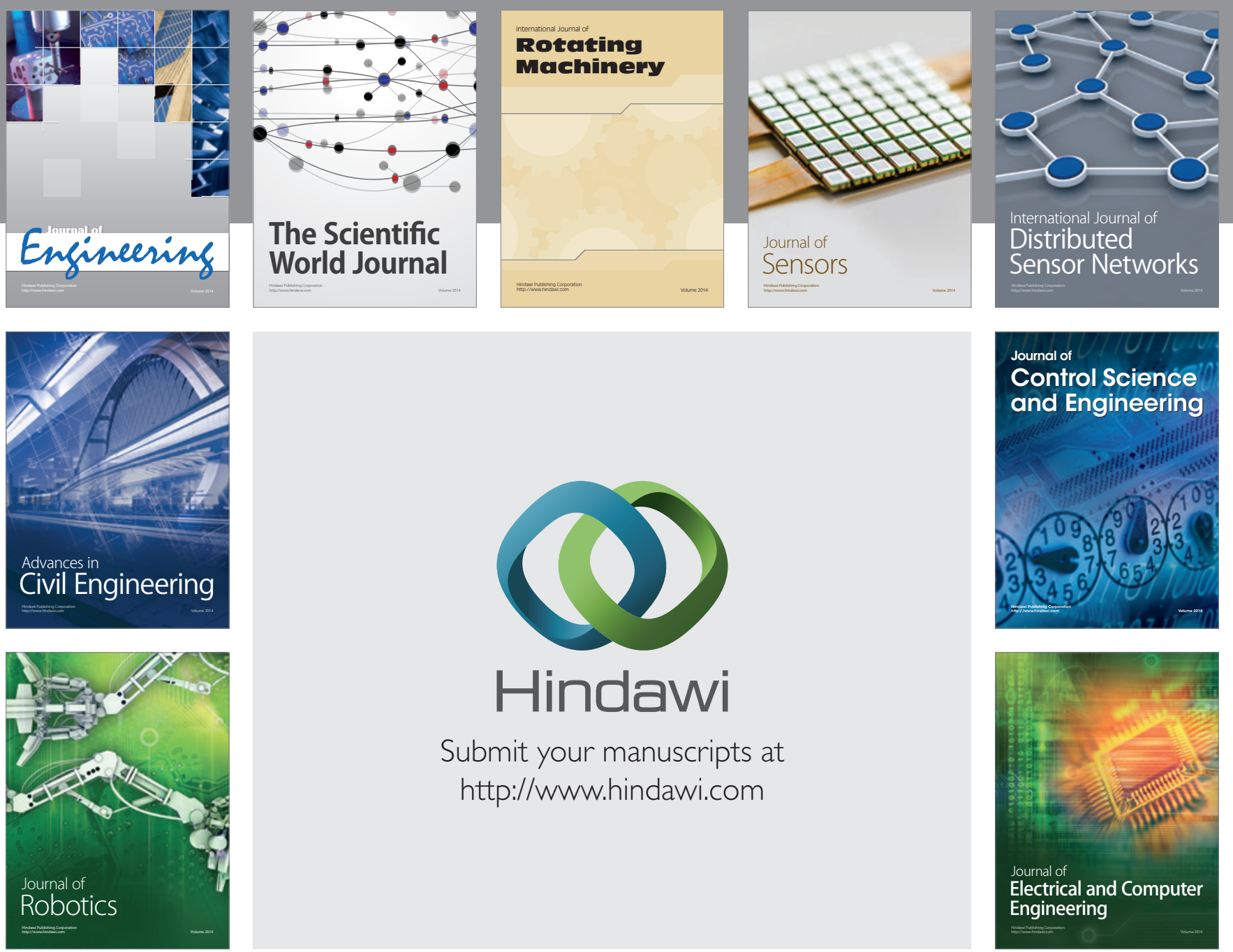

Submit your manuscripts at

http://www.hindawi.com
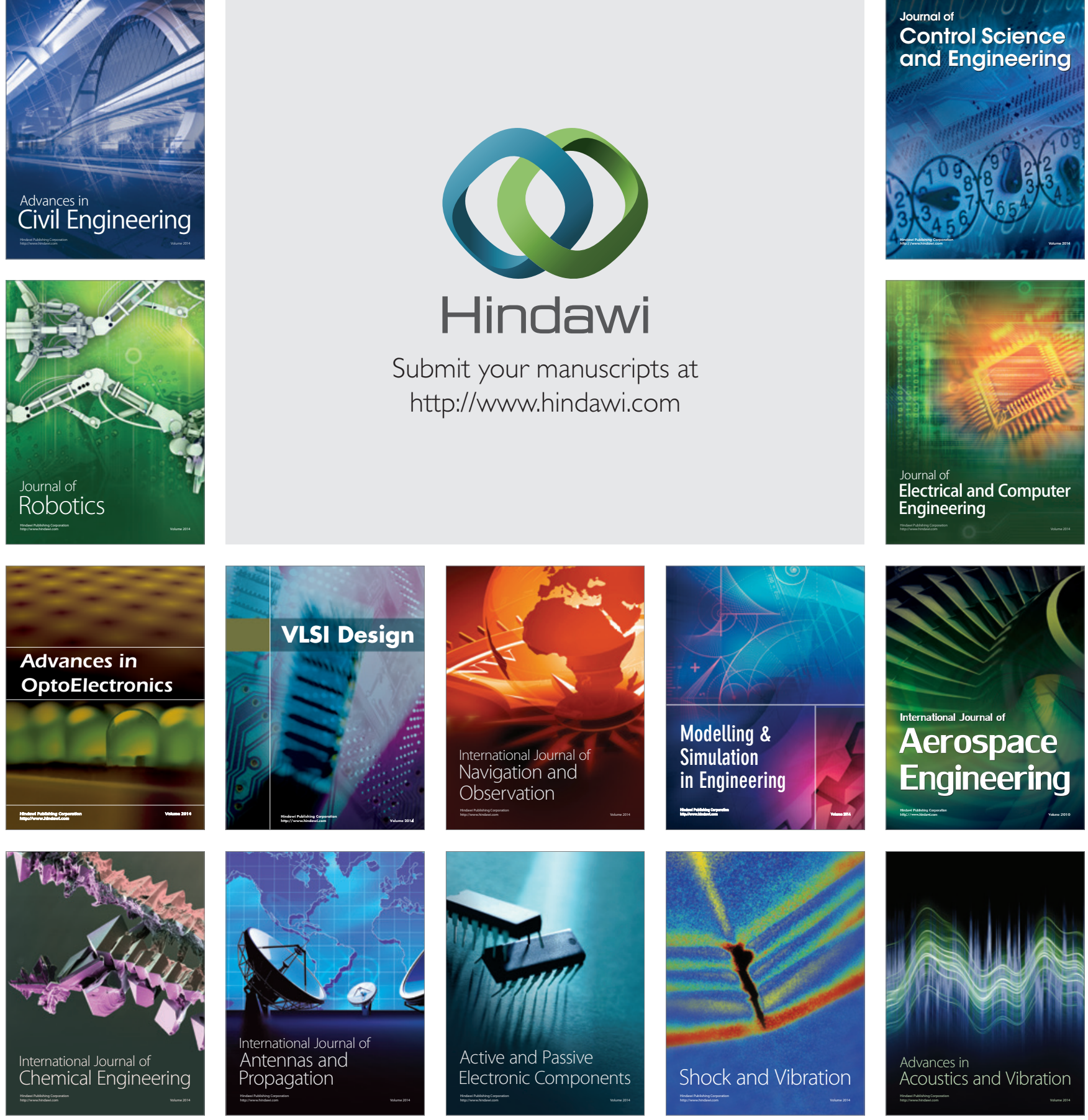